\title{
Chest pain and plaque rupture without high-sensitive troponin elevation
}

\author{
Federico Moccetti, Matthias Bossard, Richard Kobza, Florim Cuculi \\ Cardiology Division, Heart Center, Luzerner Kantonsspital, Luzern, Switzerland
}

A 41-year-old male with established coronary artery disease and previous ST-elevation myocardial infarction requiring treatment of the right coronary artery (RCA) with a drug eluting stent (DES) presented to the emergency department with acute-onset typical angina (Canadian Cardiovascular Society IV). Electrocardiogram and clinical examination were unremarkable. The values of high-sensitivity troponin $\mathrm{T}$ drawn at admission ( $4 \mathrm{~h}$ after chest pain onset) and $3 \mathrm{~h}$ later were $4 \mathrm{ng} / \mathrm{L}$ and $3 \mathrm{ng} / \mathrm{L}$, respectively $\left(99^{\text {th }}\right.$ percentile cut-off value $<14 \mathrm{ng} / \mathrm{L}$ ). Creatinine kinase-MB and myoglobin remained within normal range as well. Due to the pain characteristics and patient's past history troponin-negative acute coronary syndrome (ACS) (unstable angina) was suspected and early invasive evaluation of his coronary anatomy was undertaken. Left coronary arteries were normal, a patent DES (Fig. 1, arrow) in the proximal RCA, but a hazy-appearing region (Fig. 1, arrowhead) in the mid-RCA was noted. Since this region seemed angiographically inconclusive, an optical coherence tomography (OCT) was performed. This revealed a ruptured plaque with a rupture cavity (Fig. 1, asterisk) at the site of interest (Fig. 1, segments 1-3). The plaque rupture was interpreted as the culprit lesion and was treated with 1 DES. The patient was discharged the following day.

Despite the advent of high-sensitive troponin assays, physicians should still be aware of troponin-negative ACS presentations. As illustrated herein, acute plaque rupture accompanied by acute chest pain does not necessarily result in cardiac necrosis and elevated troponin levels. OCT represents a valuable technology to better understand the underlying pathophysiology and identify the culprit lesion(s) leading to ACS, particularly in patients with inconclusive angiograms.

Conflict of interest: None declared

Address for correspondence: Florim Cuculi, MD, Cardiology Division, Heart Center, Luzerner Kantonsspital, 6000 Luzern 16, Switzerland, tel: +41 4120521 34, e-mail: florim.cuculi@luks.ch

Received: 22.04.2018 Accepted: 7.11.2018 


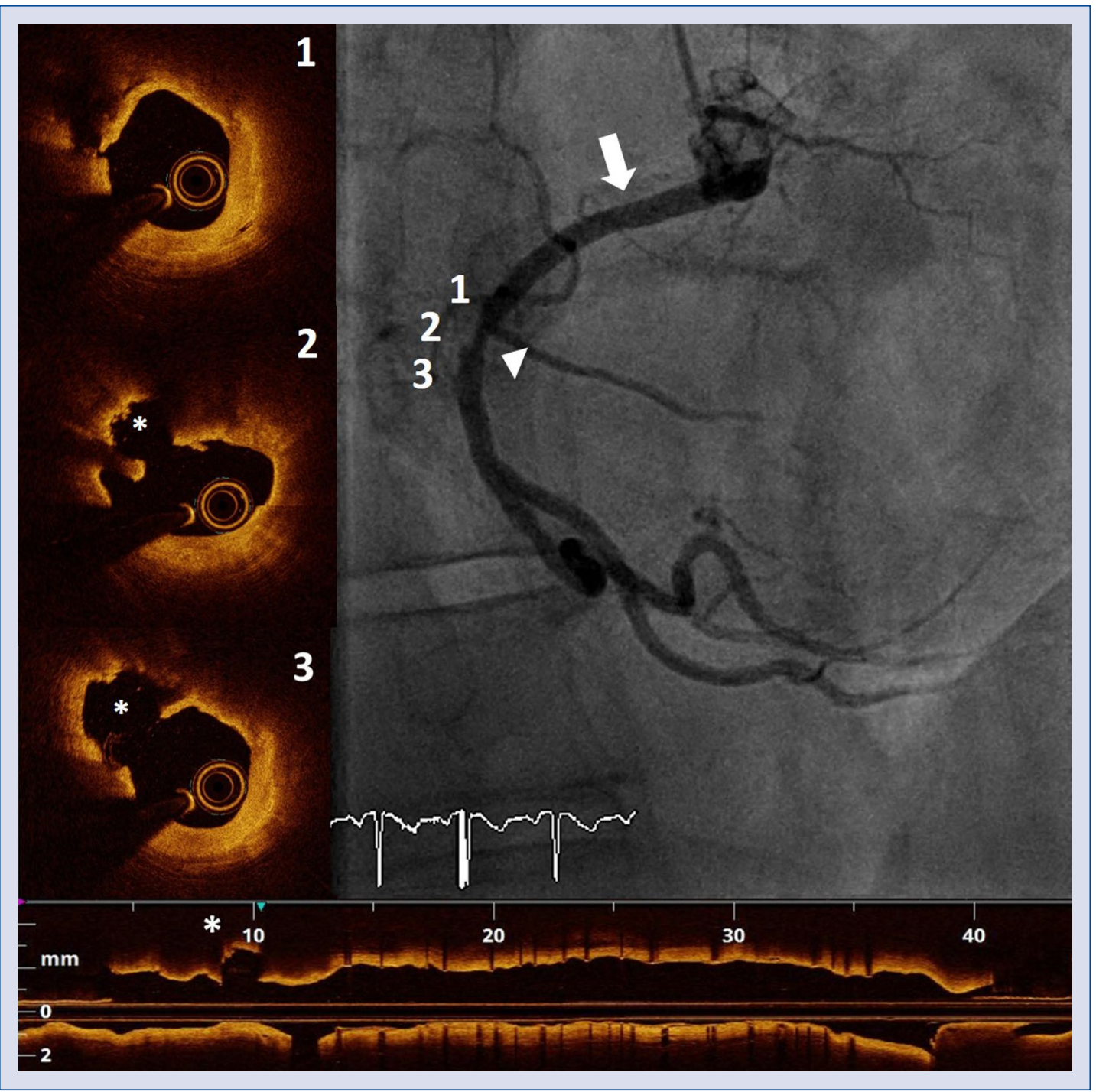

Figure 1. Right coronary artery (RCA) with previously placed drug eluting stent (arrow). Mid-RCA lesion with associated haziness (arrowhead) and corresponding frames from optical coherence tomography (OCT) pullback (1-3) showing plaque rupture and ruptured cavity $\left(^{*}\right)$. On OCT minimal luminal area at the culprit site was $3.3 \mathrm{~mm}^{2}$ and area stenosis $27 \%$. On quantitative coronary angiography minimal luminal diameter was $1.6 \mathrm{~mm}$ and degree of stenosis $44 \%$. 